\title{
Factores Asociados a la Deserción Institucional en la Universidad de la Guajira
}

\author{
Cecilia Constanza Lemos Ruiz
}

Ph.D. Métodos de Investigación y Diagnóstico en Investigación Universidad Complutense de Madrid, UCM, España.

\section{Edwin Cardeño Portela.}

Ph.D Ciencias Gerenciales Universidad Rafael Belloso Chacín, Venezuela

\section{Marcos Siosi Pino}

Psicólogo, Universidad de la Sabana Candidato a Magíster en Ciencias de la Educación, Universidad de Matanzas, Cuba.

Para citar este artículo: Lemos, C, Cardeño, E., Siosi, M.(2016). Aplicación de los modelos de respuesta binaria a los determinantes de la demanda de postgrado en Colombia. Escenarios, 14 (1), p,p19-34 DOI: http: / / dx.doi.org/10.15665/ esc.v14i1.875

Recibido: febrero 18 de 2016

Aceptado: marzo 31 de 2016

\section{RESUMEN}

La Universidad de la Guajira ha incursionado en proyectos de investigación que apuntan a caracterizar, describir y explicar razones o motivos que pueden estar asociados a variables tales como: repitencia, deserción de programa, alta permanencia, deserción institucional y demás que pueden influir negativamente frente a la orientación del ministerio de educación nacional de lograr altas tasas de retención escolar. disminuir las tasas de deserción institucional elevando el nivel de desempeño académico, es la razón fundamental por la que se ha emprendido éste y otro tipo de proyectos que permitan, a la postre, diseñar estrategias de intervención pedagógica con la activa participación tanto de los estudiantes como de los docentes de los diferentes programas académicos de la universidad, la investigación realizada pretende dilucidar los motivos con los que los estudiantes desertores explican la decisión tomada de retirarse de la institución. El estudio tomó los períodos académicos transcurridos desde el primer semestre del año 2009 hasta el primero del año 2012, consultando cuatro grandes categorías de factores: a) personales; b) académicos; c) socio-económicos y culturales, y d) institucionales, por medio de la recolección de información de fuentes secundarias y primarias, cuyos resultados se presentan a través de los análisis contenidos en este documento. Como modelo, esta investigación toma categorías del orden psicológico y sociológico, siguiendo las directrices del ministerio de educación nacional, aunque con una postura ecléctica que permita abarcar el mayor número de categorías que pueden tener influencia en la deserción institucional. el bajo desempeño académico, que proviene principalmente del nivel previo con el que ingresan a la educación superior, parece ser el mayor obstáculo para el logro de altos niveles de retención.

Palabras clave: deserción institucional, factores psicológicos, factores sociológicos, desempeño académico previo. 


\section{ABSTRACT}

The University of La Guajira, Colombia, has developed research projects that intend to characterize, describe and explain the reasons that might be associated to variables such as: repeating, retiring from a program, high permanence and institutional desertion which can influence negatively the National Educational Ministry orientations in the sense of achieving higher levels of student retention. - The fundamental reason to develop this project has been to diminish the institutional desertion while raising the level of students' academic performance. The results of the research will allow designing pedagogical intervention strategies to achieve this goal in the different programs the University has at the moment. It also intends to elucidate the motives the students say they had in order to abandon the institution. The study examined the academic periods from first semester in 2009 to the first one in 2012, consulting four categories: a) personal, b) academic, c) socio-economic and cultural, and d) institutional ones, recollecting information from primary and secondary sources, whose results were analyzed and presented in this document. As a model, the research embraces psychological and sociological categories following the Educational National Ministry guidelines, though with an eclectic position that will allow embracing the higher number of subcategories that may be influencing student's institutional retirement. The low academic performance level that may come basically from the previous school level with which students enter the University, seems to be the main problem to have high levels of students' retention.

Key words: Institutional desertion, psychological and sociological factors, previous academic performance.

\section{INTRODUCCIÓN}

La deserción ha sido considerada como la interrupción de la trayectoria académica del estudiante, que se manifiesta en la desvinculación del proceso de formación, creando una separación entre las expectativas de formación y las posibilidades reales de cumplirlas.

Puede ser clasificada en tres grupos: a) según su duración, temporal o definitiva; b) según su alcance, del programa curricular pero no de la institución; de la institución, pero no del sistema de la educación superior; finalmente, del sistema educativo, y c) según el mecanismo, por norma institucional o voluntariamente (UNAL, 2005).

Según González (2005), la deserción se define también como el abandono, voluntario u obligado de la carrera en la que se matricula un estudiante. Algunas definiciones más prácticas dicen que la deserción en la educación superior se refiere al número de estudiantes que abandona el sistema entre uno y otro período académico (semestre o año) y se calcula como el balance entre la matrícula total del primer período, menos los egresados del mismo período, más los alumnos reintegrados en el período siguiente, que produce el nuevo estado de los alumnos matriculados sin deserción.

Páramo y Correa (2000, p. 4), hablan de varios tipos de deserción que no son excluyentes entre sí: a) deserción total o abandono definitivo de la formación académica; b) discriminada por causas; c) deserción por facultad o cambio de facultad; d) deserción por programa o cambio de programa en una misma facultad; e) deserción en el primer semestre de la carrera por inadaptación a la vida universitaria; f) deserción acumulada o la sumatoria de la deserción en una institución, considerándola a su vez como una decisión personal que no obedece necesariamente a un retiro forzoso.

Sánchez, Navarro y García (2002) plantean que la deserción se puede entender como el "retiro forzoso o voluntario que el estudiante hace de un programa académico o de la universidad" (p. 2), forzoso cuando por mortalidad académica el estudiante se ve obligado a retirarse de la institución. Entre los factores que la pueden 
causar mencionan los: a) personales, constituidos por motivos psicológicos (motivacionales, emocionales, desadaptación, insatisfacción); o sociológicos por influencias familiares, de amistades, compañeros; edad, salud, etc.; b) académicos, dados por problemas cognitivos, bajo desempeño académico, repitencia, inadecuados métodos de estudio, inadecuadas metodologías de enseñanza, inadecuada infraestructura física, espacios y equipamiento para el estudio, falta de orientación vocacional y profesional, poca aptitud hacia la academia, entre otros; c) socioeconómicos, producto de los bajos ingresos familiares, el desempleo, la falta de apoyo del grupo familiar, incompatibilidad de horario de trabajo y estudio, etc.; y d) institucionales, problemas de ineficiencia administrativa, programas obsoletos que no cumplen las expectativas, nivel bajo en la calidad de la educación.

La Universidad de Chile (2008) realizó un estudio buscando las causas de la deserción fundamentando su teoría principalmente sobre dos autores considerados como los precursores de enfoques teóricos sobre la calidad de la educación: Austin y Tinto. El primero señalaba que la retención del estudiante en la institución dependía en gran medida del nivel en que se involucraba tanto en las relaciones con los docentes como con los demás estudiantes y con la misma institución. Tinto, por su parte, plantea que la decisión de permanecer o no en la institución depende de varias variables, tales como nivel académico previo, falta de claridad vocacional, nivel socio económico, compromiso del estudiante, expectativas personales, etc. Algunos factores institucionales pueden también influir en la decisión de desertar, pero de nuevo este factor depende del involucramiento que haya desarrollado el estudiante con los elementos que constituyen la institución, incluida la comunidad educativa.

El tema de la deserción se tornó de interés universal a partir de las tasas históricas de deserción que se observaron en la década de los sesenta, en países de la Organización para la Cooperación y el Desarrollo Económico (OCDE) con sede en Bélgica, las cuales oscilaban entre el $30 \%$ y $50 \%$ (p. 41). La retención de los estudiantes, en cambio, se conforma como un indicador de buen desempeño, de cumplimiento de las metas de formar estudiantes hasta graduarlos y por ende, de calidad.

Cabrera, L., Tomás, J., Álvarez, P. y González, M. (2006, p. 3), la consideran como el abandono de los estudios o interrupción antes de finalizarlos, incluyendo en esta categoría factores tales como: a) abandono involuntario; b) abandono de la carrera para iniciar otra en la misma institución; c) abandono de la carrera para iniciar otra en otra institución; d) dejar la universidad e irse a otra para completar estudios iniciados; e) renunciar a la formación universitaria para iniciar itinerarios formativos fuera de la universidad, o incorporarse al mundo laboral; f) interrumpir la formación con la intención de retomarla en el futuro; y otras posibilidades.

En el estudio realizado en Uniguajira recopilado bajo el título: La permanencia del estudiante de Uniguajira: de las cifras a la realidad académica (Lemos, C; Cardeño, E; Redondo, M; Deluque, L. (en prensa), se examinaron las variables de repitencia, deserción de programa y alta permanencia, teniendo claro que las dos primeras conducen a la tercera. De las inferencias realizadas en la construcción de las conclusiones, se observó que el nivel previo con el que acceden los estudiantes a la educación superior es una de las principales razones de repitencia y probablemente también del cambio del programa inicial (p. 106), convirtiéndose así el nivel de desempeño académico en el primer motivo que acarrean tales dificultades. Aunque las condiciones económicas también aparecen como un factor de predisposición, no es la primera causa, y más bien los hábitos de estudio junto a los escasos espacios extracurriculares de apoyo al estudiante por parte del profesor, además del despliegue de metodologías tradicionales, se evidencian como factores de peso. Igualmente, se observó que la falta de orientación vocacional en los niveles previos no permite al estudiante elegir una carrera que convoque sus capacidades y satisfaga las expectativas de estudio y laborales.

Peralta (2008), recurre a Tinto para definir en la teoría y en la práctica el concepto de deserción como "una situación a la que se enfrenta un estudiante cuando aspira y no logra concluir su proyecto educativo" (p. 4), tomando como de- 
serción la inactividad académica durante tres semestres consecutivos, conocida por algunos como primera deserción, después de la cual el estudiante podría eventualmente retomar el programa, cambiar de programa o no regresar. Cita a González como quien propone distinguir entre "dos tipos de abandono (...), con respecto al tiempo (inicial, temprana y tardía) y con respecto al espacio (institucional, interna y del sistema educativo)"' (p. 5). Para Tinto, según Peralta, hay períodos que se podrían considerar críticos en la deserción; a) durante la admisión, por la imagen que a los estudiantes les puede haber llegado a través de diversos medios y que pueden haber creado falsas expectativas; lo ideal, dice el autor, es que la institución imparta una imagen realista de lo que es y ofrece; b) en el primer semestre, se pueden presentar dificultades porque el mundo del colegio puede diferir mucho del universitario, ya que este último es más impersonal; igualmente el cambio se sectores rurales a urbanos; el tamaño de la institución y muchos otros a los que el nuevo estudiante debe acostumbrarse.

Por su parte, el Ministerio de Educación Nacional (2009) reconoce que no hay todavía un concepto preciso que defina la deserción estudiantil; sin embargo, existe un consenso que la define como el abandono de la educación, explicado a través de diferentes categorías de variables: individuales y personales, socioeconómicas, académicas e institucionales. Obedezca el abandono a una u otra categoría, desde el punto de vista institucional, el estudiante que abandona "crea un lugar vacante en el conjunto estudiantil que pudo haber sido ocupado por otro alumno que persistiera en los estudios" (p. 20), causando cuando menos problemas financieros a las instituciones al producir inestabilidad en la fuentes de ingresos; además del posible incumplimiento de la políticas y metas sociales establecidas por el Estado. Por otra parte, la deserción también genera grandes costos sociales asociados a la pérdida de la productividad laboral, por una menor acumulación de capital humano evidenciada en los diferentes niveles de ingresos que pueden lograr los profesionales frente a los que no llegan a serlo.

Citando a Tinto y a Giovagnoli, el MEN conjuga las definiciones de estos dos autores para establecer la deserción como:
Una situación a la que se enfrenta un estudiante cuando aspira y no logra concluir su proyecto educativo, considerándose como desertor a aquel individuo que siendo estudiante de una institución de educación superior no presenta actividad académica durante dos semestres académicos consecutivos, lo cual equivale a un año de inactividad académica (p. 22).

Asimismo, el MEN define dos tipos de abandono estudiantil: respecto al tiempo y respecto al espacio. Con respecto al tiempo la clasifica en: a) precoz, refiriéndose a aquel estudiante que habiendo sido admitido no se matricula; b) temprana, se refiere al estudiante que abandona los estudios en los primeros semestres; y c) tardía, el estudiante que abandona en los últimos semestres.

Con respecto al espacio, la divide en: a) deserción institucional, cuando el estudiante abandona la institución, y b) deserción del programa o interna, cuando el estudiante se cambia a otro programa que ofrece la misma institución.

Himmel (2002) citando el modelo de Fishbein y Ajzen, señala que la decisión de desertar está influida por conductas previas, que llevan a un "debilitamiento de las intenciones iniciales" (p. 97) o, según Ethington, por el contrario, a "la perseverancia, la elección y el desempeño" (p. 98).

Paralelamente surgen los modelos sociológicos, con Spady como uno de los autores más conocidos, quién se basa en la teoría del suicido de Durkheim que contempla la deserción como un "resultado de la ruptura de un individuo con el sistema social, por su imposibilidad de integrarse a la sociedad, sugiriendo, por tanto, que la deserción obedece a la falta de integración de los estudiantes con el entorno de la educación superior. Por otro lado, considera que el factor económico puede influir positivamente si el estudiante percibe que los ingresos de un egresado de la educación superior son mejores que los que se derivan de otra actividad alterna no profesional.

Tinto expande el modelo de Spady incorporando la teoría del intercambio de Nye (p. 101), quién plantea que los seres humanos "evitan las con- 
ductas que implican algún costo para ellos" ( $p$. 101), lo cual implica para Tinto que la retención o la deserción de un estudiante es el "resultado de un proceso longitudinal de interacciones con los sistemas académico y social de la universidad" (p. 101).

Finalmente, el proyecto: Determinación de los factores asociados a la deserción institucional de los estudiantes de la Universidad de La Guajira, da un paso más en esta temática, con el propósito fundamental de proporcionar datos e información veraz que permita a la institución diseñar estrategias de intervención para bajar las tasas de deserción, elevando, a la vez, el nivel de desempeño académico, siendo de esta manera también consecuentes con los hallazgos analizados en los estudios anteriores.

\section{Aspectos Metodológicos}

Desde posturas teóricas como la del Ministerio de Educación Nacional (2009), se asume que la deserción en la educación superior, es un elemento que hace parte del conjunto del sistema educativo y de la vida misma universitaria, existiendo a lo largo de su historia con momentos más o menos críticos, como se señalaba en la sección anterior, ya que está relacionado con los procesos siempre dinámicos y cambiantes por los que transita el estudiante desde el momento de su admisión. Se atribuye su causa, acorde al SPADIES , a varios factores a) factores personales; b) factores académicos; c) factores socio-económicos y culturales, y d) factores institucionales. La Fuente secundaria la conforman los documentos elaborados a partir de los datos archivados en la Oficina de Admisiones y Registro de la Universidad de la Guajira Las fuentes primarias las constituyen 93 desertores de los diferentes programas de la institución, quienes fueron localizados y entrevistados, gracias a los datos recogidos de las fuentes secundarias.

El período que se determinó fue de 7 semestres académicos, superando los 3 semestres que plantean algunos de los autores examinados (Peralta, 2008), dado que según algunos de ellos (Tinto citado por Peralta, 2008), en tan corto período de tiempo el estudiante puede tomar la decisión de reincorporarse a la institución en el mismo programa o ingresar a otro programa, lo cual ya no lo haría un desertor institucional.

\section{Informe de Trabajo de Campo}

Después de este breve recorrido por los modelos más destacados con los cuales se ha examinado y explicado el fenómeno de la deserción, esta investigación toma tanto categorías del orden psicológico como sociológico, siguiendo, en parte, las directrices del Ministerio de Educación Nacional, a la vez que tomando una posición ecléctica que permita abarcar el mayor número de categorías que pueden tener influencia en la deserción institucional.

El estudio también, consulta las cuatro categorías, ya mencionadas: a) factores personales; b) factores académicos; c) factores socio-económicos y culturales, y d) factores institucionales.

En el trabajo de campo se revisaron los desertores de todos los programas por cohorte iniciada durante en el primer semestre académico de 2009, quienes al finalizar el primer semestre de 2012 deberían haber terminado el séptimo semestre académico. Siguiendo las orientaciones del MEN, todo estudiante que en este período de tiempo, no se haya matriculado durante tres semestres consecutivos, se considera desertor. El consolidado de las cifras de deserción, elaborado a partir de los datos hallados en las fuentes secundarias, aparece en la tabla a continuación.

Como se observa, el porcentaje más alto de desertores lo tiene la Facultad de Ingeniería, seguido de la Facultad de Ciencias Económicas y Administrativas, la Escuela Técnica, la Facultad de Ciencias Sociales y Humanas, y con los porcentajes más bajos aparecen las Facultades de Ciencias Básicas y de Ciencias de la Educación, mostrando un total de $20 \%$ de desertores institucionales durante el período de tiempo estudiado.

Cabe resaltar que en el estudio de las variables de repitencia, deserción de programas y alta permanencia (Lemos et al, 2012, p. 87) (en prensa), desarrollado por la Universidad de La Guajira y el Ministerio de Educación Nacional, se observó que la mayoría de los desertores de programa eran de la Facultad de Ingeniería, conformándo- 
Figura 1. Factores determinantes de la demanda por educación

\begin{tabular}{|c|c|c|c|c|c|c|c|}
\hline \multicolumn{8}{|c|}{ ÍNDICE DE MATRÍCULA Y DESERCIÓN ENTRE 2009 I Y 2012 I EN UNIGUAJIRA } \\
\hline $\begin{array}{r}\text { FACULTAD } \\
\text { CATEGORÍA }\end{array}$ & $\begin{array}{c}\text { FACULTAD DE } \\
\text { CIENCIAS } \\
\text { ECONÓMICAS Y } \\
\text { ADMINISTRATIVAS }\end{array}$ & $\begin{array}{l}\text { CENCIAS } \\
\text { BÁSICAS }\end{array}$ & $\begin{array}{c}\text { TÉCNICAS Y } \\
\text { TECNOLÓGICAS }\end{array}$ & INGENIERÍA & $\begin{array}{c}\text { CIENCIAS } \\
\text { DE LA } \\
\text { EDUCACIÓN }\end{array}$ & \begin{tabular}{|c|} 
CIENCIAS \\
SOCIALES \\
Y \\
HUMANAS \\
\end{tabular} & $\begin{array}{c}\text { TOTAL } \\
\text { UNIGUAJIRA }\end{array}$ \\
\hline DESERCIÓN & 4241 & 83 & 820 & 3772 & 940 & 1090 & 10946 \\
\hline MATRÍCULA & 17145 & 943 & 5130 & 13268 & 10170 & 8695 & 55351 \\
\hline PORCENTAJE & 25 & 9 & 16 & 28 & 9 & 13 & 20 \\
\hline
\end{tabular}

Fuente: Elaboración propia

se en el $88,37 \%$. Igualmente, en la variable de repitencia, también esta Facultad ocupó el primer lugar, dado que los cursos en las áreas de las matemáticas y afines, fueron los que mostraron mayores índices de repitencia.

Como resultado de un proceso de conversatorios se construyó un cuestionario escalado que consta de 98 reactivos (anexo), el cual fue respondido por 93 desertores contactados a través de entrevista personal e Internet. De éstos, 48 fueron hombres y 45 mujeres.

Cuatro expertos revisaron el instrumento y, posteriormente, se hizo un estudio piloto con 30 de los participantes, con el objetivo de realizar el análisis de confiabilidad a través del Alfa de Cronbach, cuyo resultado fue de 0,842 , lo que permitió continuar con el estudio (anexo).

Durante el conversatorio con los estudiantes en repitencia y deserción de programas, se evidenció que el factor académico tenía un peso considerable entre las categorías en juego, de tal manera que el instrumento se construyó teniendo en cuenta las anotaciones obtenidas a partir de esta fuente, de la revisión bibliográfica y de estudios previos desarrollados en el tema y temas afines.

En el marco del enfoque mixto, ya en 2006, Hernández, R.; Fernández, C.; Baptista, P., (p. 755 y 759) postulaban que éste "logra una perspectiva más amplia del fenómeno (...) más integral, completa y holística, el tipo de investigación fue un "Diseño de dos etapas", por la trasformación de una clase de datos en otra, también llamado en la quinta versión "Diseño exploratorio secuencial" (Hernández et al, 2010, p. 564), pues permite matizar datos cuantitativos y cualitativos, de tal manera que sea útil en temas de proponer, diseñar y desarrollar estrategias de mejoramiento del problema detectado. Posteriormente, en la sexta versión de 2014, estos autores decantan como una de las características que representan este enfoque, "la integración y discusión conjunta de los datos cuantitativos y cualitativos para realizar las inferencias" (p 580).

\section{Discusión y Análisis de los Resultados}

La discusión y el análisis de los resultados se

Tabla 3.2 Conformación del instrumento de valoraciones escalado, por categorías, dimensiones y número de reactivos

\begin{tabular}{|l|c|c|c|c|}
\hline \multicolumn{1}{|c|}{ CATEGORIAS } & PERSONALES & ACADEMICAS & $\begin{array}{c}\text { SOCIO- } \\
\text { ECONÓMICAS } \\
\text { CULTURALES }\end{array}$ & INSTITUCIONALES \\
\hline PSICOLOGICAS & 22 & 22 & & 4 \\
\hline SOCIOLOGICAS & & 13 & 23 & 14 \\
\hline $\begin{array}{l}\text { SUBTOTALES } \\
\text { REACTIVOS }\end{array}$ & $\mathbf{2 2}$ & $\mathbf{3 5}$ & $\mathbf{2 3}$ & 18 \\
\hline
\end{tabular}


presentan por el orden de las categorías señaladas: personales, académicas, socio-económicas y culturales e institucionales.

Figura 4.1 Distribución de los desertores participantes según el rango de edad

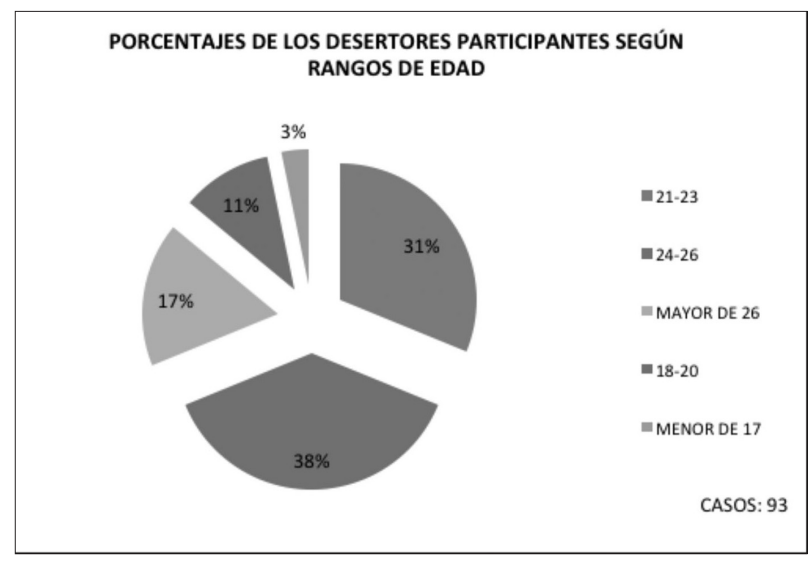

En el estudio realizado predominaron los desertores de 24 a 26 años de edad; seguido de los de 21 a 23 años; un porcentaje también alto lo constituyen los mayores de 26, mientras que los menores de 17 años apenas hacen un 3\% del total. Lo anterior se explica ya que se tomaron los desertores de al menos 3 semestres consecutivos, en un período de 3 años y medio de la misma cohorte.

\section{Factores Personales Asociados A La Deserción Institucional}

Los factores personales que pueden influir sobre la decisión que toma el estudiante de desertar, están en la dimensión del campo psicológico. El cuestionario consta de 22 reactivos en la categoría personales.

Los factores personales combinados con los académicos muestran en este estudio cómo, dependiendo de las facultades, los estudiantes están satisfechos o no con la carrera que están cursando, lo cual hace evidente que las expectativas sobre el programa escogido quizás eran otras, aunque también sugiere un bajo nivel de orientación profesional previa al ingreso a la educación superior. Pero, en cambio, manifiestan que las competencias estaban en concordancia con los requerimientos del programa; en otras palabras, parece evidente que son conscientes que las competencias o capacidades y el desempeño académico no son, necesariamente, coincidentes.

Factores tales como la inconformidad con el programa elegido, la no concordancia con las expectativas iniciales de la carrera, tienen que ver, entre otros, con "motivos psicológicos como son los motivacionales, emocionales, desadaptación, insatisfacción, etc.", que plantean Sánchez et al

Figura 4.2 Curva de factores personales asociados a la deserción institucional en Uniguajira, por facultades.

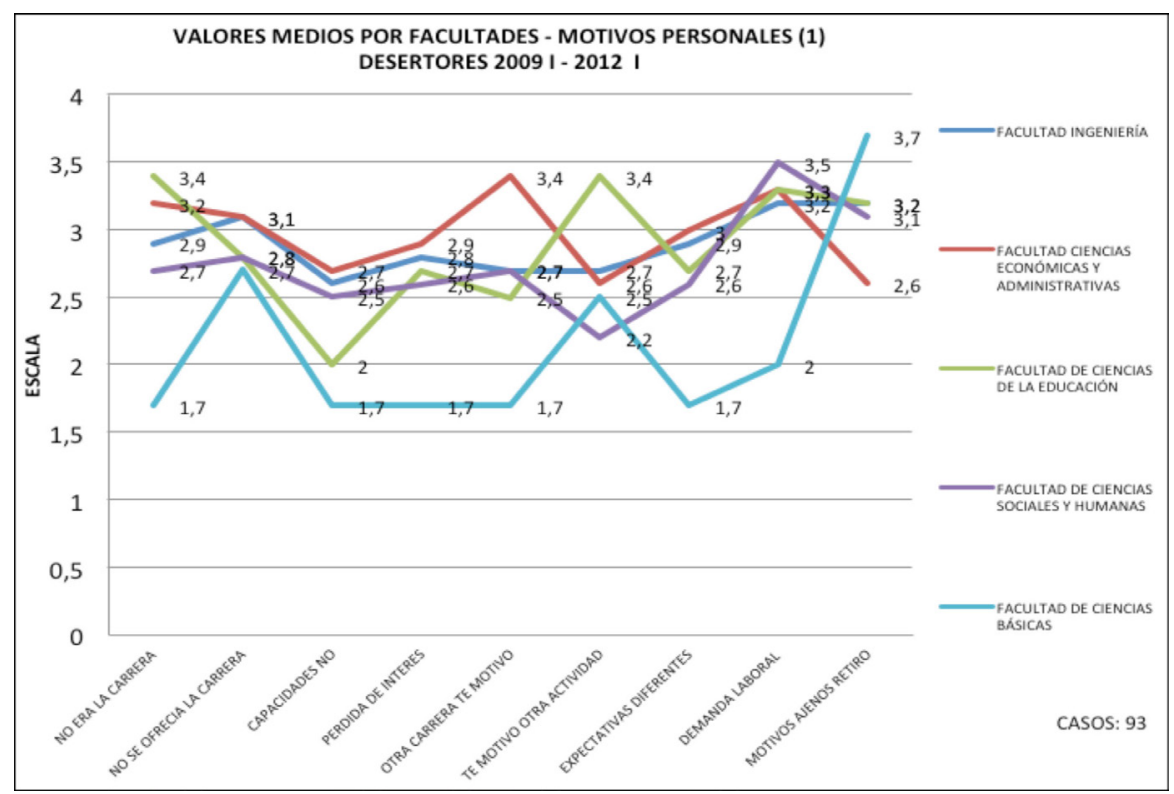


en la fundamentación teórica del presente documento, enmarcados en los aspectos personales que en el análisis parecen haber influido en el retiro de la institución. Así mismo, la falta de orientación vocacional y profesional, citada por la Universidad de Chile, la poca aptitud hacia la academia, por ejemplo, que, aunque se ubican en el factor académico, tienen igualmente un tinte personal, ya que en este tema, dichos factores, parecen estar estrechamente articulados.

Por otra parte, reconocen que el débil método de estudio no les ayuda superar los obstáculos académicos encontrados en el paso de la educación media a la superior, puesto que son conscientes de tener tiempo suficiente para estudiar, tema que trata también Sánchez et al, al señalar como factores académicos los "inadecuados métodos de estudio".

En la dimensión de lo personal, el tema de salud es también mencionado por Sánchez et al como un factor sociológico que puede incidir en el abandono de los estudios; en el estudio aparecen el embarazo o el matrimonio como factores que tuvieron influencia en la decisión de retiro.

Factores Académicos Asociados A La Deserción Institucional

Tanto en la literatura revisada como en los estu- dios realizados por la Universidad en temas afines, se ha observado que el factor académico es muy significativo a la hora de tomar la decisión de desertar; además de los casos de retiro forzoso o normativo que toma la institución (retiro por asuntos disciplinarios, etc.,) que también ocurren.

En todas las facultades y en anteriores estudios ha quedado de manifiesto que problemas como los de repitencia, los cuales, en ocasiones pueden llevar a la deserción del programa, tienen como una de los principales factores de ocurrencia, el bajo nivel académico con el que ingresa el estudiante a la Universidad.

Al indagar sobre la influencia que pudo haber tenido la preparación previa que recibieron en la educación media en la decisión de retirarse de la Universidad, varios grupos de desertores hacen referencia a esta situación, alegando que el paso más rápido en la Universidad se suma a las debilidades que traían haciendo muy difícil "ponerse al día" en los asuntos académicos, de tal manera que cada vez se alejan más de la posibilidad de éxito.

La Universidad de Chile citando a Tinto plantea que entre las variables que influyen en el abandono se encuentran el nivel académico previo, la falta de claridad vocacional, etc. Debido a estos y

Figura 4.3 Curva de factores personales asociados a la deserción institucional en Uniguajira, por facultades.

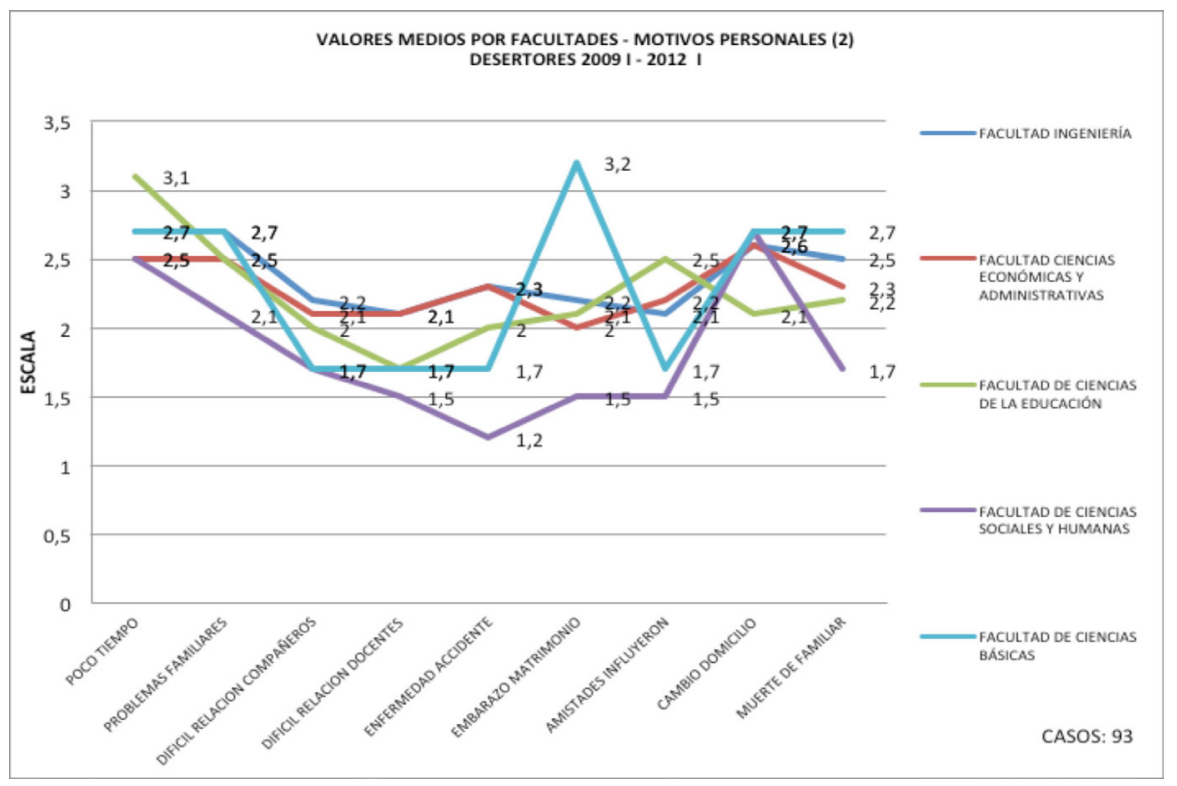


Figura 4.4 Valores medios por Facultades de factores académicos asociados a la deserción institucional en Uniguajira

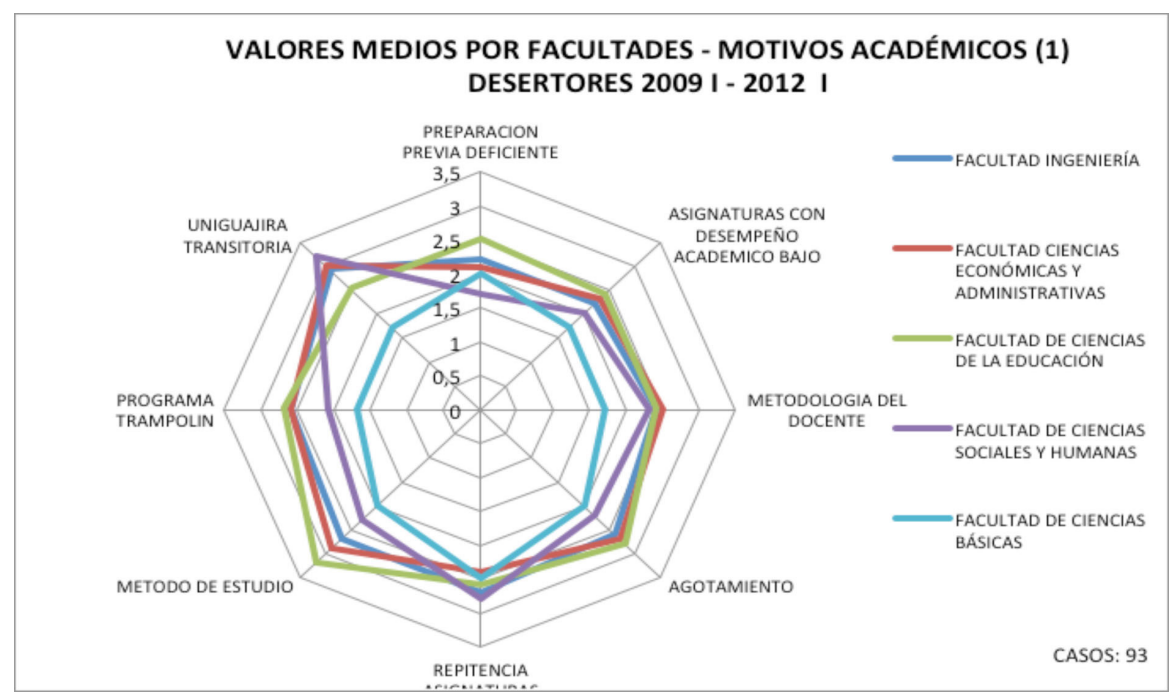

otros hallazgos, Uniguajira ha planteado un proyecto de intervención para el mejoramiento del desempeño académico del estudiante, articulando los campos del pensamiento lógico matemático y la lengua española, para tratar de superar las dificultades que más se han manifestado entre la población de repitentes y desertores.

Parece ser que, como lo apunta Sánchez et al, también la existencia de metodologías inadecuadas de enseñanza, el poco apoyo extracurricular por parte de los docentes, la exigencia académica universitaria en comparación con lo vivido en el bachillerato, pueden conducir al desánimo y a la toma de decisión de abandonar $\mathrm{y}$, aunque las mayores tasas de repitencia se han producido en los campos de las matemáticas y afines, los participantes de las diferentes facultades manifiestan que la repitencia y el bajo nivel de comprensión de los contenidos influye en el deseo de abandono.

Así mismo, señalan la apatía en el desarrollo de sus actividades por fuera de clase como una de las causas que, una vez los ha conducido al bajo desempeño académico, también los puede inducir al retiro, ya sea del programa o institucional, denotando que tienen conciencia de que sus limitaciones académicas y posiciones actitudinales pueden jugar buena parte del peso para el abandono.

En cuanto al sistema de evaluación, hubo estu- diantes que dicen no haber encontrado un equilibrio entre el nivel de comprensión de los contenidos curriculares y los procesos evaluativos, lo que posteriormente los llevó a tomar la decisión de desertar. Igualmente, señalan tanto el nivel de complejidad de las evaluaciones como la discontinuidad en los periodos evaluativos como factores pre disponentes para tomar la decisión de retiro.

Es de resaltar cómo los desertores de las facultades participantes toman diferentes direcciones en las valoraciones en torno a las evaluaciones, de tal manera que la diversidad de tipos de evaluaciones fue un factor incidente en dos facultades, mientras que el continuo proceso evaluativo al que eran sometidos por parte de los docentes fue un factor impulsador de la deserción en otras dos facultades.

Algunos cursos que aparentemente no tenían ninguna relación con la carrera elegida fue el factor decisivo para el retiro según los estudiantes de las facultades de Ciencias Económicas y Administrativas, lo mismo que para los de Ciencias Sociales.

Definitivamente no era la carrera de su preferencia y por ello decidieron retirarse de la universidad manifestaron los estudiantes de las facultades de Ciencias Administrativas y económicas y los de Ciencias de la Educación. 
En síntesis, los motivos que influyen en el abandono de los estudios por los factores académicos, parecen divergir de acuerdo con el programa, por lo tanto, se observa una gran diversidad de valoraciones, mientras a los desertores de un programa los afecta un aspecto a otros los afecta uno diferente; pero de todas maneras, el factor académico parece ser uno de los más influyentes en el abandono de la institución.

\section{Factores Socio-Económicos Y Culturales Aso- ciados A La Deserción Institucional}

Los factores socio-económicos y culturales también se contemplan en el estudio con una participación de 23 reactivos.

En todas las facultades y en anteriores estudios ha quedado de manifiesto que problemas como los de repitencia, los cuales, en ocasiones pueden llevar a la deserción del programa, tienen como una de los principales factores de ocurrencia, el bajo nivel académico con el que ingresa el estudiante a la Universidad.

Al indagar sobre la influencia que pudo haber tenido la preparación previa que recibieron en la educación media en la decisión de retirarse de la Universidad, varios grupos de desertores hacen referencia a esta situación, alegando que el paso más rápido en la Universidad se suma a las debilidades que traían haciendo muy difícil "ponerse al día" en los asuntos académicos, de tal manera que cada vez se alejan más de la posibilidad de éxito.

La Universidad de Chile citando a Tinto plantea que entre las variables que influyen en el abandono se encuentran el nivel académico previo, la falta de claridad vocacional, etc. Debido a estos y otros hallazgos, Uniguajira ha planteado un proyecto de intervención para el mejoramiento del desempeño académico del estudiante, articulando los campos del pensamiento lógico matemático y la lengua española, para tratar de superar las dificultades que más se han manifestado entre la población de repitentes y desertores.

Parece ser que, como lo apunta Sánchez et al, también la existencia de metodologías inadecuadas de enseñanza, el poco apoyo extracurricular por parte de los docentes, la exigencia académica universitaria en comparación con lo vivido en el bachillerato, pueden conducir al desánimo y a la toma de decisión de abandonar $\mathrm{y}$, aunque las mayores tasas de repitencia se han producido en los campos de las matemáticas y afines, los participantes de las diferentes facultades manifiestan que la repitencia y el bajo nivel de comprensión de los contenidos influye en el deseo de abandono.

Así mismo, señalan la apatía en el desarrollo de sus actividades por fuera de clase como una de las causas que, una vez los ha conducido al bajo desempeño académico, también los puede inducir al retiro, ya sea del programa o institucional, denotando que tienen conciencia de que sus limitaciones académicas y posiciones actitudinales pueden jugar buena parte del peso para el abandono.

En cuanto al sistema de evaluación, hubo estudiantes que dicen no haber encontrado un equilibrio entre el nivel de comprensión de los contenidos curriculares y los procesos evaluativos, lo que posteriormente los llevó a tomar la decisión de desertar. Igualmente, señalan tanto el nivel de complejidad de las evaluaciones como la discontinuidad en los periodos evaluativos como factores pre disponentes para tomar la decisión de retiro.

Es de resaltar cómo los desertores de las facultades participantes toman diferentes direcciones en las valoraciones en torno a las evaluaciones, de tal manera que la diversidad de tipos de evaluaciones fue un factor incidente en dos facultades, mientras que el continuo proceso evaluativo al que eran sometidos por parte de los docentes fue un factor impulsador de la deserción en otras dos facultades.

Algunos cursos que aparentemente no tenían ninguna relación con la carrera elegida fue el factor decisivo para el retiro según los estudiantes de las facultades de Ciencias Económicas y Administrativas, lo mismo que para los de Ciencias Sociales.

Definitivamente no era la carrera de su preferencia y por ello decidieron retirarse de la universidad manifestaron los estudiantes de las facultades de Ciencias Administrativas y económicas y 
Figura 4.5 Valores medios por Facultades de factores socio-económicos y culturales asociados a la deserción institucional en Uniguajira

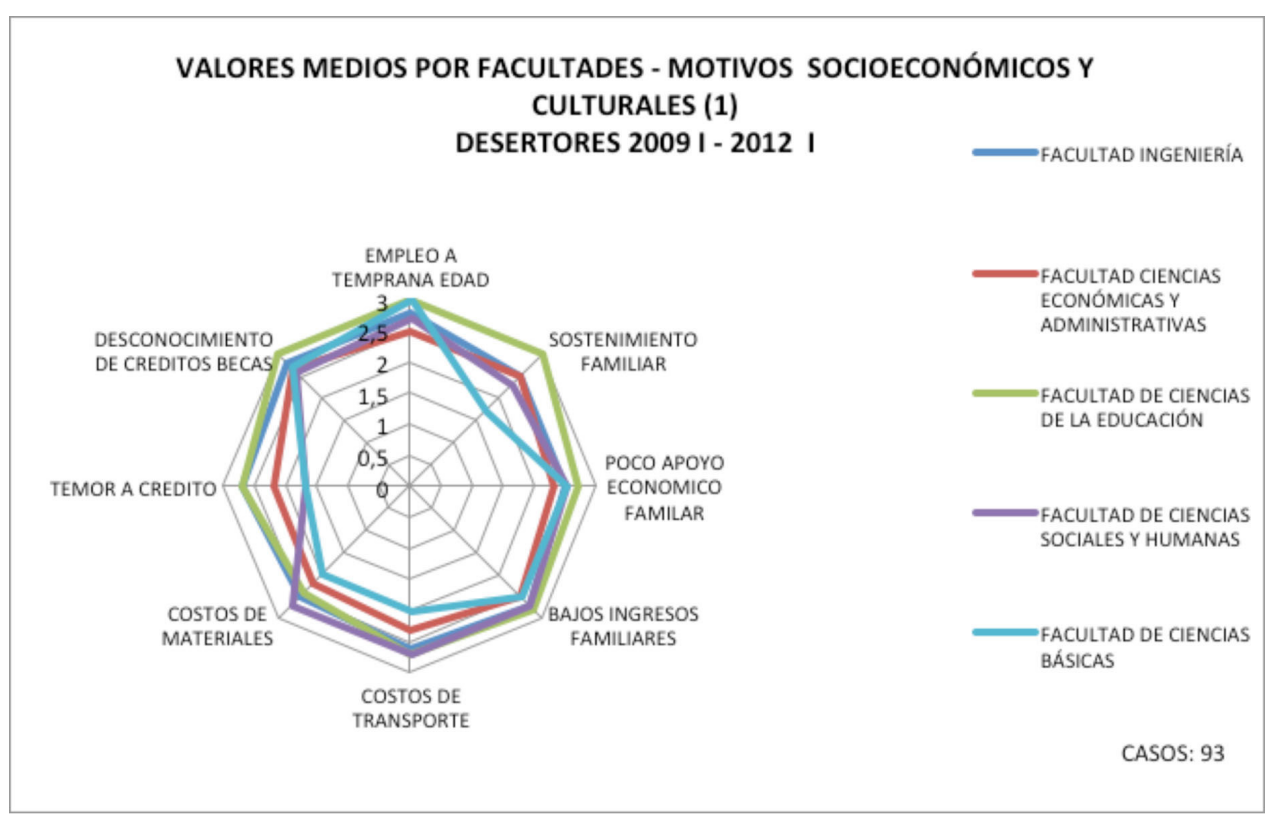

los de Ciencias de la Educación.

En síntesis, los motivos que influyen en el abandono de los estudios por los factores académicos, parecen divergir de acuerdo con el programa, por lo tanto, se observa una gran diversidad de valoraciones, mientras a los desertores de un programa los afecta un aspecto a otros los afecta uno diferente; pero de todas maneras, el factor académico parece ser uno de los más influyentes en el abandono de la institución.

\section{Factores Socio-Económicos Y Culturales Aso- ciados A La Deserción Institucional}

Los factores socio-económicos y culturales también se contemplan en el estudio con una participación de 23 reactivos.

Las bajas valoraciones dadas en este estudio confirman cómo el factor de índole económico no es el prioritario para los desertores al tomar la decisión de abandonar la carrera. Cabe resaltar que tanto los estudiantes en repitencia como en deserción de programa, y ahora los desertores institucionales o no son conscientes del peso que el factor económico puede tener sobre los problemas de permanencia en la educación superior, o no desean manifestarlo así.

Sin embargo, a pesar de reconocer el poco apoyo financiero familiar para estudiar, la mayoría de los respondientes consideran que estudiar es un asunto prioritario en la familia. Algunos de ellos creen que la falta de equipos, conexión a Internet y no contar con una buena bibliografía en el hogar puede haber influido en el abandono; sin embargo, aunque el estudio pertenece al factor socio económico y cultural, se presume que éstos están igualmente relacionados en el académico de tal manera que el efecto se manifiesta en un bajo desempeño.

\section{Factores Institucionales Asociados a la Deser- ción Institucional}

La situación en que se halla la institución repercute también en la motivación que tienen los desertores en la toma de la decisión; aunque, igualmente, la institución cumpliendo la normatividad, puede exigir el retiro forzoso del estudiante.

Se observa, como lo manifiesta Tinto, citado antes, que la información que ofrece la Universidad, a través de los diferentes medios, puede estar creando falsas expectativas en lo que respecta al plan dele estudio y al programa. Parece que existe una brecha entre lo que algunos aspirantes piensan acerca de la profesión a la que ingresan y el programa que ofrece la Universidad, 
Figura 4.6 Valores medios por Facultades de factores socio-económicos y culturales asociados a la deserción institucional en Uniguajira

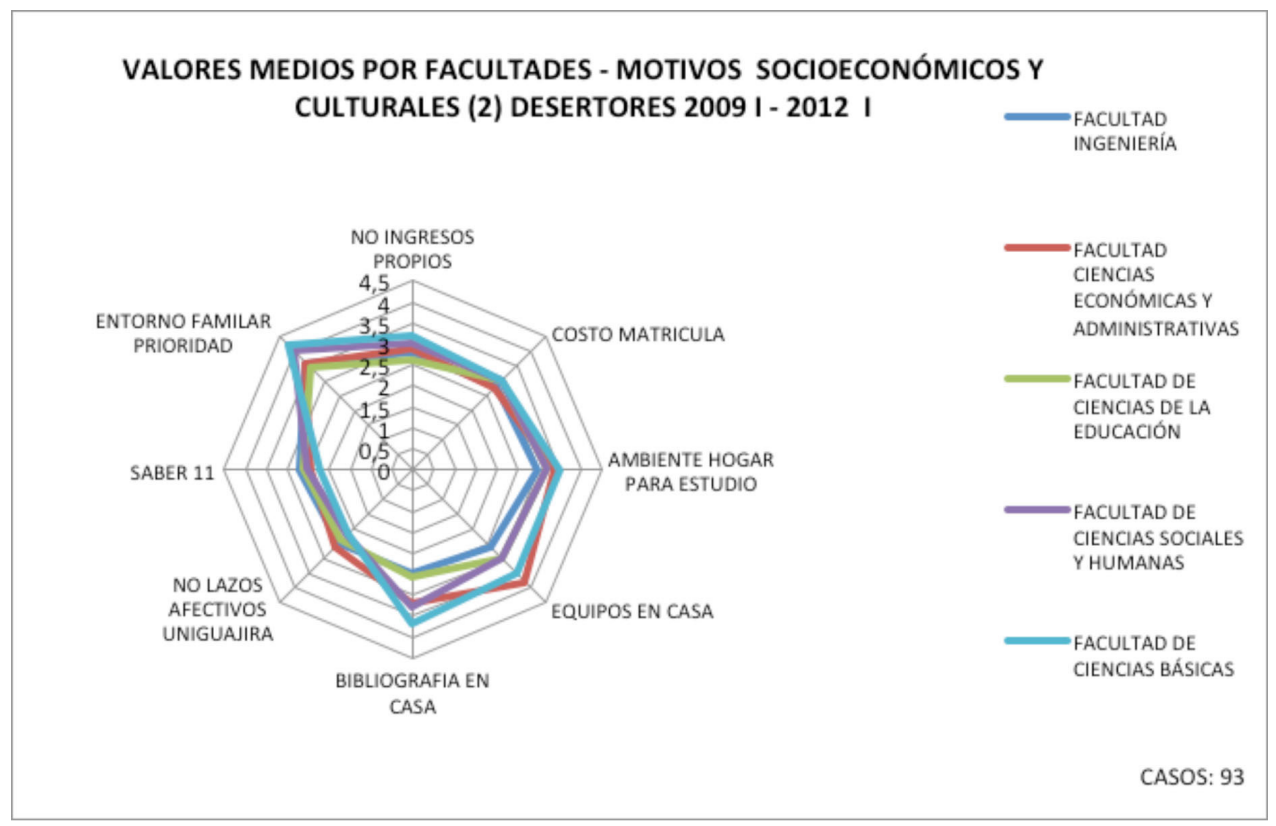

Figura 4.7 Valores medios por Facultades de factores institucionales asociados a la deserción institucional en Uniguajira

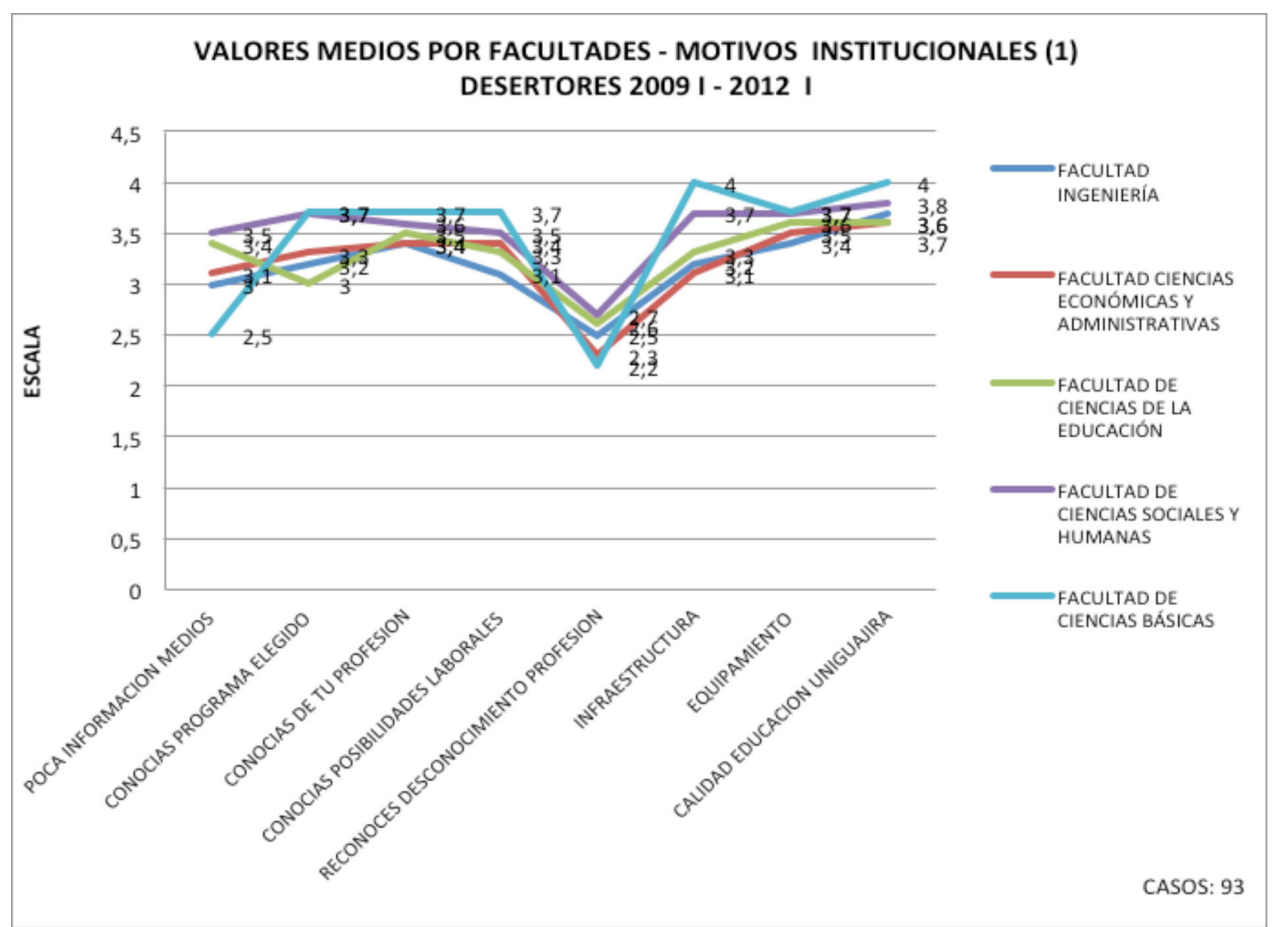


o, en otras palabras, no coinciden las expectativas con la realidad. La infraestructura no alcanzó niveles satisfactorios para todos los respondientes, pero en cambio en el nivel de la calidad de la educación hubo consenso satisfactorio.

\section{Análisis De Los Datos De Respuesta No Esca- lada, Cerrada O De Múltiple Escogencia}

Se ha observado que algunas veces el hecho de tener que dejar la casa para vivir en otra ciudad donde se va a estudiar, causa dificultades de adaptación que pueden también influir en la decisión de retirarse y volver al hogar. En la investigación, 35 de los participantes tuvieron que dejar la casa para estudiar; la mayoría piensan que dejar la casa les causó dificultades de adaptación que influyeron en la decisión de retirarse.

23 de los desertores dicen haber venido de estudios técnicos o tecnológicos previos, pero en su gran mayoría expresan que esto no les causó dificultades que hubieran influido en la decisión de dejar la Universidad.

Figura 4.8 Distribución de los desertores participantes según el estrato social

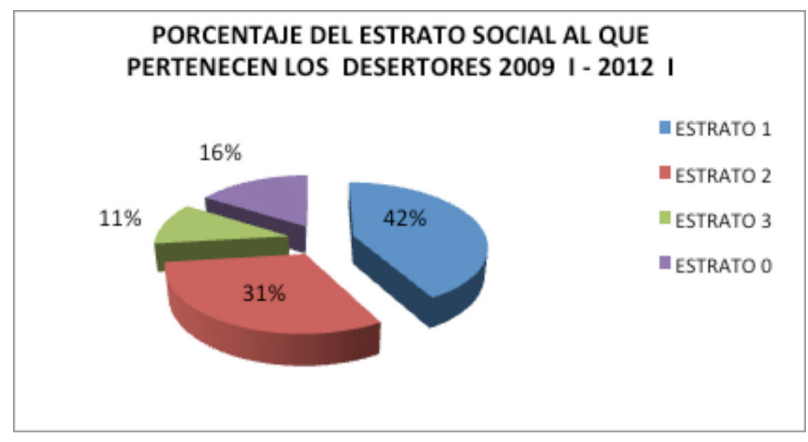

Las universidades estatales y en particular la Universidad de La Guajira, recibe estudiantes que generalmente pertenecen a los estratos sociales más bajos, quienes por lo general han egresado de planteles en categorías bajo, inferior y muy inferior según las categorías del ICFES, particularidad que también tiene implicaciones de orden académico.

La figura muestra cómo la mayoría de los desertores se ubican en los estratos 1 y 2 , seguido del estrato 0 (particularmente para las poblaciones indígenas). La Universidad cuenta aproxi- madamente con un 52\% de estudiantes indígenas, principalmente de la etnia Wayuu, quienes cuentan con beneficios en el valor de las matrículas, transporte y demás, bajo el propósito es incluirlos y retenerlos en la educación superior.

Figura 4.9 Distribución de las preferencias por las actividades de fin de semana

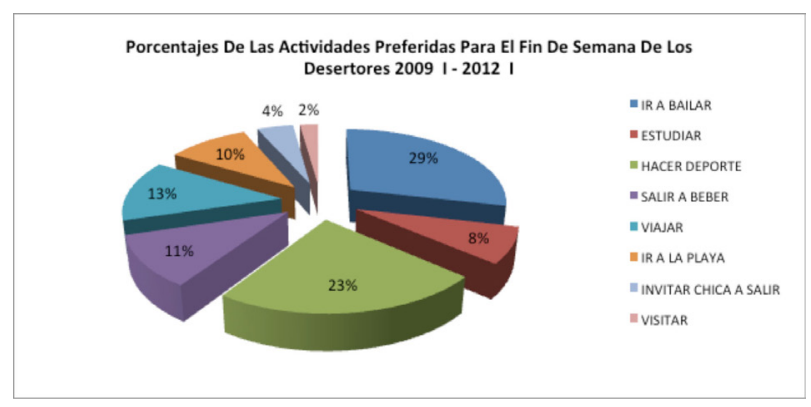

Las actividades de fin de semana preferidas por los desertores participantes como se observa, son en su orden: ir a bailar, hacer deporte, viajar, salir a tomarse unas copas, ir de playa, etc.; el renglón de estudiar como una actividad preferida para un fin de semana ocupa el sexto lugar entre ocho oportunidades de escogencia. Esta escogencia coincide con las consideraciones que los estudiantes en repitencia y deserción de programas hacían en conversatorios previos, en los cuales reconocían que le dedicaban poco tiempo al estudio; igualmente, la literatura revisada para la construcción d este documento (Sánchez, Navarro y García, 2002) tiene como uno de los factores del bajo desempeño, el poco compromiso del estudiante con el estudio, además de los hábitos incorrectos con los que realizan dicha actividad.

Figura 4.10 Nivel educativo de los padres de los desertores participantes

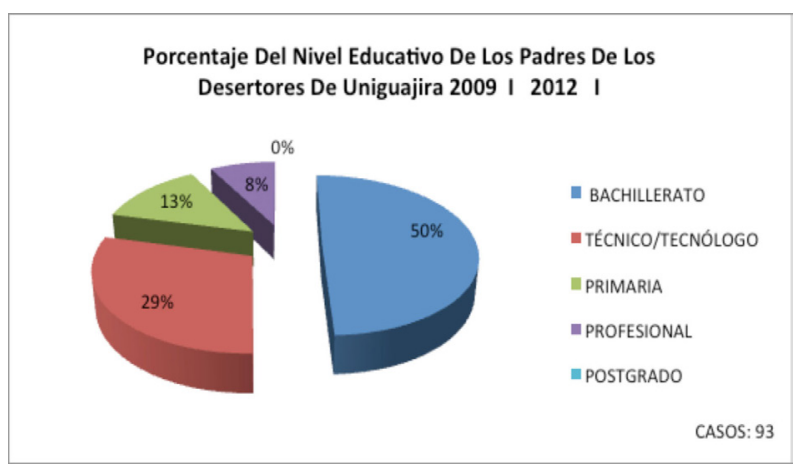


La distribución del nivel educativo de los padres de los desertores parece tener que ver con la importancia que se le da a la actividad de estudiar en el entorno familiar, frente a otras actividades, generalmente laborales. En este caso, la mitad de los padres de los participantes tiene bachillerato completo, un alto porcentaje dice que su padre es técnico o tecnólogo, un pequeño porcentaje de los padres de los participantes son profesionales y no se registran padres con título de postgrado. En el estudio Diagnóstico vocacional de los bachilleres 2007 de Maicao (2011), se observó que las aspiraciones de los estudiantes para ingresar a la educación superior e incluso para estudiar una carrera profesional versus una técnica o tecnológica, estaba relacionada con el nivel educativo de los padres, principalmente con el de la madre; en otras palabras, a mayor nivel profesional de los padres, mayores aspiraciones de los hijos.

Figura 4.11 Nivel educativo de las madres de los desertores participantes

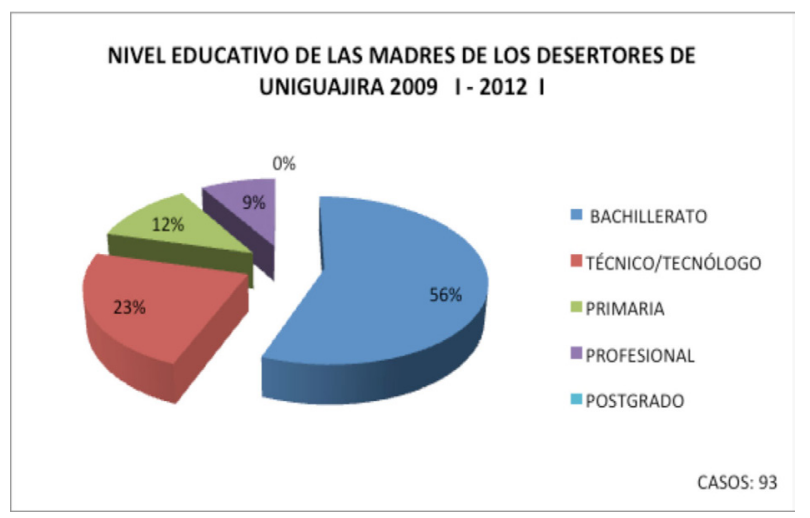

Hay mayor número de madres con bachillerato completo, pero menor número de técnicas o tecnólogas. También hay un leve incremento entre las madres profesionales que entre los padres, aunque tampoco hay madres de los participantes, postgraduadas.

A los participantes se les pidió que mencionaran las actividades que les hubieran ayudado a tener mejores resultados en las pruebas SABER 11, siendo el simulacro, la preparación o las prácticas de la pruebas las que señalaron en su mayoría; en segundo lugar, aparece mejores hábitos de estudio y en tercero, la lectura.

Cuando se les solicita dar un lugar a la Universidad en el Departamento de La Guajira por la importancia que tiene comparada con otras entidades e instituciones, la mayoría le dan el segundo lugar (38); sin embargo, hay un número también alto que le da un cuarto lugar (25). En cuanto a la imagen que tiene Uniguajira como institución académica en el medio, la respuesta de la mayoría (58) de los participantes es que tiene una buena imagen.

Figura 4.12 Valoración que dan los desertores 2009 I - 2012 I, de elementos del sistema de Uniguajira

Distribución De La Valoración Que Los Desertores 2009 I2012 I Dan A Los Elementos Del Sistema De Uniguajira

$=$ DOCENTES $=$ ESTUDIANTES $=$ DIRECTIVOS $=$ ADMINISTRATIVOS $=$ PLANTA FÍSICA

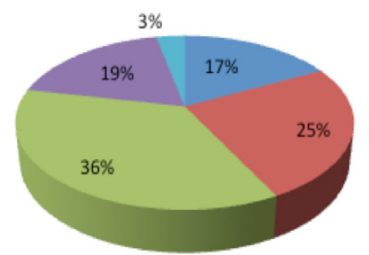

CASOS: 93

Para los participantes, los directivos ocupan la primera posición en el orden de importancia que tienen los elementos que componen el sistema educativo de Uniguajira, y esto se debe a que, según ellos, de este grupo dependen las políticas y su operacionalización en el escenario universitario. Asuntos como los beneficios en las matrículas, la apertura de nuevos programas, la calidad de la educación, el estado de la planta física y de los equipos, la vinculación de los docentes, etc., están ligados a las decisiones que se tomen en los círculos jerárquicos de la institución.

Los estudiantes ocupan el segundo lugar, como centro de la educación y principales usuarios del servicio. Sin embargo, los docentes están después de los administrativos, lo que puede denotar que la calidad del servicio que presta la institución depende no solamente de los directivos, como grupo que toma las decisiones, sino también de la planta de personal administrativo 
que las operativiza.

Figura 4.13 Distribución de los elementos del sistema de Uniguajira que deben mejorar según los desertores 2009 I - 2012 I

Cuando se les pide enumerar los elementos que debe mejorar la Universidad, se concentran en la planta física, particularmente en los salones de clase $y$, en segundo lugar, en el sistema de matrículas. Igualmente, señalan el transporte como uno de los factores que se debe optimizar.

\section{Conclusiones}

A partir de los anteriores análisis se puede inferir que:

En el factor personal hay desertores quienes consideran que la Universidad de La Guajira no ofrece una carrera que realmente llene sus expectativas. Igualmente, hay algunos que consideran que fue otra carrera lo que los motivó a dejar la inicial, lo cual hace eco de la premisa anterior. Aunque la consideración es personal, lo anterior se articula con lo académico, lo socio cultural y también con lo institucional, ya que el ofrecimiento de una diversidad de programas tiene que ver en algún sentido con cada una de estas categorías.

Por otra parte, para algunos de los desertores, actividades diferentes al estudio influyeron en el abandono del programa; además de que también tenían otras expectativas sobre el programa al que se matricularon, entre ellas la creencia de que la carrera tenía una mejor demanda laboral.

En cuanto al factor académico, es el bajo desempeño el principal motivo de deserción, pues produce un desánimo que al no poder ver superado conduce a la decisión de abandono.

En el factor socio-económico y cultural no se observan consideraciones significativas; además, en su mayoría, conceptúan que el estudio era una prioridad en el entorno familiar.

Finalmente, en el factor institucional, consideran que la Universidad debe proveer a la sociedad con mayor información y más precisa sobre los programas que ofrece, sus contenidos, posibilidades laborales, etc., aunque en términos generales, no consideran el factor institucional como relevante al momento de tomar la decisión de desertar.

\section{Recomendaciones}

- La oferta de nuevos programas en la Universidad puede convertirse en una posibilidad para muchos estudiantes y desertores, cuyas expectativas de estudio eran diferentes a las ofrecidas en la institución, además de satisfacer otras necesidades sociales educativas.

- La institución debería establecer una divulgación periódica, veraz y detallada sobre los programas que ofrece, desde la página Web de la Universidad y otros medios, que llegara permanentemente a la ciudadanía en general.

- El ofrecimiento de apoyo al estudiante desde el momento de su ingreso, en los campos del conocimiento que guarden relación con el plan de estudios que va a cursar en la formación profesional, podría convertirse en una estrategia temprana para salirle al paso a la situación académica previa del estudiante que se manifiesta en el bajo desempeño, principalmente en los primeros semestres de la carrera.

- La operacionalización de un registro semestral de los estudiantes que solicitan retiro temporal, indagando los motivos por los cuales toma la decisión, para mantener contacto con el estudiante, facilitando mantener una información actualizada que permita tomar decisiones adecuadas de intervención temprana.

\section{Referencias}

Cabrera, L., Tomás, J., Álvarez, P. y González, M. (2006). El problema del abandono de los estudios univer-sitarios. RELIEVE, v. 12, n. 2, p. 171203.

http: / / www.uv.es / RELIEVE / v12n2 / RELIEVEv12n2_1.htm

Donoso, S.; Shiefelbein, E. (2007). Análisis de los modelos explicativos de la retención de estudiantes en la universidad: una visión desde la desigualdad social. En: Estudios Pedagógicos XXXIII, No. 1:7-27. Documento PDF, 22 p.

Hernández, R.; Fernández, c.; Baptista, P. (2006). 
Metodología de la investigación. Cuarta edición. McGrawHill. México. 850 p.

(2010). Metodología de la investigación. Quinta edición. McGrawHill. México. 613 p.

(2014). Metodología de la investigación. Quinta edición. McGrawHill. México. 600 p.

Himmel, Erika K. (2002). Modelos de análisis de la deserción estudiantil en la educación superior. Retención y movilidad estudiantil. En: Calidad en la Educación Núm.:17 Págs.:91-108 Biblioteca: ANUIES.

Lemos, C.; Cardeño, E.; Redondo, M.; Deluque, L. La permanencia del estudiante de Uniguajira: de las cifras a la realidad académica. (2012). 103 p.

Ministerio de Educación Nacional (2009). Deserción estudiantil en la educación superior colombiana. Metodología de seguimiento, diagnóstico y elementos para su prevención. Documento PDF. 158 p.
Páramo, G.; Correa, C. (2000). Deserción estudiantil universitaria. Conceptualización. Documento PDF. 14 p.

Peralta, C. (2008). Modelo conceptual para la deserción estudiantil universitaria chilena. Universidad Católica de la Santísima Concepción. Estudios Pedagógicos XXXIV, $\mathrm{N}^{\circ}$ 2: 65-86. Versión On-line ISSN 0718-0705.

Sánchez, G.; Navarro, W.; García, A. (2002). Factores de la deserción estudiantil en la Universidad Surcolombiana. Facultad de Educación. Universidad Surcolombiana. Documento PDF. $7 \mathrm{p}$.

Universidad de Chile (2008). Departamento de Economía. Centro de Microdatos. Documento PDF. 143 p.

Universidad Pedagógica Nacional. La deserción estudiantil: reto investigativo y estratégico asumido de forma integral por la UPN. (SF). Documento PDF. 10 p. 